\title{
A Population Analysis of the Initial SARS-CoV-2 Outbreak in Chicago: Implications for the Future
}

\author{
Ikechi Ekezie, MCOptom ${ }^{1} \bullet$ Ayobamidele A. Tiamiyu, MD²
}

ABSTRACT: Objectives. We set out to examine the initial pattern of SARS-CoV-2 infection among different racial and ethnic groups in a large multiracial city in the United States and investigate the implications for containing possible future outbreaks.

Design. Cross-sectional study.

Methods. We accessed racially stratified data released daily by the Chicago Department of Public Health and analyzed the ethic distribution of SARS-CoV-2 infections and fatalities over a 30-day period at the outset of the SARSCoV-2 outbreak in Chicago. We computed the point prevalence and the cause-specific mortality rates for White, Hispanic, and African Americans to compare infection and death rates among Hispanics and African Americans relative to Whites.

Results. We found that the initial SARS-CoV-2 infection rates among Hispanics and African Americans, respectively, were 1.59 and 2.38 times the infection rate among the White population in Chicago. The corresponding death rates for Hispanics and African Americans, respectively, were 1.29 and 3.65 times the death rate in the White population. When infection and death rates for African Americans vs Whites were analyzed (using Mann-Whitney $U$ testing), the $P$ value was $<.0001$ for both measures, demonstrating statistical significance. Comparing these same measures for Hispanic vs Whites, neither the infection rates $(P=.1091)$ nor death rates $(P=.3817)$ reached a level of statistical significance.

Conclusion. During the initial outbreak of SARS-CoV-2, Hispanic and African American Chicagoans were infected and died at greater rates than Whites. Multiple factors likely account for these differences; however, controlling these factors could have the effect of mitigating the initial impact of the disease in the most affected population groups and reducing the overall magnitude of the disease in the United States.

\section{AFFILIATIONS:}

${ }^{1} \mathrm{NHS}$ Grampian, Aberdeen, Scotland, United Kingdom

${ }^{2}$ All Saints University School of Medicine, Roseau, Dominica

\section{CITATION:}

Ekezie I, Tiamiyu AA. A population analysis of the initial SARS-CoV-2 outbreak in Chicago: implications for the future. Consultant. Published online September 21, 2020. doi:10.25270/con.2020.09.00010

Received July 6, 2020. Accepted August 7, 2020.

\section{DISCLOSURES:}

The authors report no relevant financial relationships.

\section{CORRESPONDENCE:}

Ikechi Ekezie, MCOptom, 4525 W School St, Chicago, IL 60641 (ike.ekezie@nhs.net)

\section{$\mathrm{T}$} he 2019 SARS-CoV-2 outbreak was initially identified on December 12, 2019, in Wuhan, China, ${ }^{1,2}$ and has rapidly spread around the world. ${ }^{3}$ The first case of SARS-CoV-2 infection in the United States was reported on January 20, 2020. ${ }^{4}$ As of September 9, 2020, more than 6.3 million people in the United States had been infected, and more than 189,000 had died. ${ }^{5}$ The United States now has the highest number of infections and deaths from SARS-CoV-2 in the world. ${ }^{6}$ In Chicago, Illinois, the first case was reported on January 24, 2020. ${ }^{7}$ As of September 9, 2020, more than 73,000 residents of Chicago had been infected with the virus, and more than 2800 patients had died from the disease. ${ }^{8}$

Chicago is the third-largest US city based on gross domestic product. ${ }^{9}$ It comprises an ethnically diverse population of 


\begin{tabular}{|c|c|c|}
\hline & Number & $\%$ of Total \\
\hline Male & 12,757 & 50.7 \\
\hline Female & 12,400 & 49.3 \\
\hline Total & 25,157 & 100.0 \\
\hline \multicolumn{3}{|c|}{ Confirmed COVID-19 Cases $^{\mathrm{a}}$} \\
\hline White & 3303 & 13.13 \\
\hline African American & 6820 & 27.11 \\
\hline Hispanic & 6729 & 26.75 \\
\hline Other ${ }^{\mathrm{b}}$ & 8305 & 33.01 \\
\hline Total & 25,157 & 100 \\
\hline \multicolumn{3}{|c|}{ Confirmed COVID-19 Deaths } \\
\hline White & 183 & 17.36 \\
\hline African American & 532 & 50.47 \\
\hline Hispanic & 249 & 23.62 \\
\hline Other $^{b}$ & 90 & 8.54 \\
\hline Total & 1054 & 100 \\
\hline \multicolumn{3}{|c|}{$\begin{array}{l}\text { a Patients whose diagnosis was under investigation were not included } \\
\text { in these data. } \\
{ }^{\circ} \text { This group comprised Asian patients and other non-Hispanic } \\
\text { patients. }\end{array}$} \\
\hline
\end{tabular}

approximately 2.7 million people and is $32.8 \%$ White, $30.1 \%$ Black or African American, 29.0\% Hispanic or Latino, and $6.4 \%$ Asian; $1.7 \%$ of the population comprises other ethnicities. ${ }^{10}$

In order to assess the early impact of SARS-CoV-2 on the 3 major racial communities in Chicago, we analyzed SARS-CoV-2 data from publicly available records and computed infection and death statistics for White, Hispanic, and African American Chicagoans.

\section{METHODS}

We collated and analyzed daily SARS-CoV-2 data released online by the City of Chicago Department of Public Health (CDPH) from April 5, 2020, and May 4, 2020 (Table 1). ${ }^{11}$ These data detail the daily cumulative total number of infections and deaths within the city and include information on patients' ages, gender, ethnicity, and the presence or absence of underlying conditions. Patients whose diagnosis was listed as "under investigation" were excluded from the data.

To compare infection and death rates between different ethnic and racial groups, we began by calculating the daily cause-specific mortality rate (CSMR) and daily point prevalence (PP) for
White, Hispanic, and African American patients.

The CSMR is the number of deaths assigned to a specific cause during a given time interval divided by the mid-interval population. The CSMR was determined for White, Hispanic, and African-American patients with COVID-19 by dividing the relevant daily cumulative number of deaths by the corresponding 2018 Chicago population estimate; this was done separately for non-Hispanic Whites, African Americans, and Hispanics. At the end of the study period, a cumulative mean CSMR was also computed.

PP is the number of current cases of a disease (new and pre-existing) at a specified point in time divided by population at the same specified point in time. To calculate the PP, we used the daily cumulative infection numbers for each ethnic group and divided this figure by that group's 2018 population. A mean PP was also computed at the end of the study period.

The total population of Chicago and the White, Hispanic, and African American populations of the city used in these calculations were derived from the US Census Bureau's 2018 population estimate for Chicago, ${ }^{10}$ in which the total population was $2,705,994$, the White population was 887,566 , the African American population was 814,504 , and the Hispanic population was 784,738 .

Our data were tabulated in an Excel spreadsheet. Statistical analysis of the daily cumulative data was done using GraphPad Prism software, version 8.4.2.679. To test for differences between data from White patients and data from minority (Hispanic and African American) patients, we performed Mann-Whitney U tests on the daily cumulative PP and CSMR figures for Whites vs African Americans and for Whites vs Hispanics.

\section{RESULTS}

$P P$. Our analysis showed that the ratio of the mean PP for Hispanic patients to the mean PP for White patients was 1.59. The ratio of the mean PP for African American patients to the mean PP for White patients was 2.38. Mann-Whitney U tests of the difference between the daily cumulative PP data for White patients vs the daily PP data for Hispanic patients was not statistically significant $(P=.1091)$; for White patients vs African American patients, this difference was statistically significant $(P<.0001)$.

CSMR. The ratio of the mean CSMR for Hispanic patients to the mean CSMR for White patients was 1.29. The ratio of the mean CSMR for African American patients to the mean CSMR for White patients was 3.65. Mann-Whitney U tests of the difference between the daily cumulative CSMR data for White patients vs the daily cumulative CSMR data for Hispanic patients was not statistically significant $(P=.3817)$; for White patients vs African American patients, this difference was statistically significant $(P<.0001)$. The results of this statistical analysis are presented in Table 2. 


\section{A Population Analysis of the Initial SARS-CoV-2 Outbreak in Chicago: Implications for the Future}

\section{Table 2. Statistical Analysis}

\section{Mean PP and CSMR Data}

\begin{tabular}{|c|c|}
\hline \multicolumn{2}{|l|}{$\operatorname{PP}\left(\times 10^{-1}\right)$} \\
\hline Mean PP White & $22.17(95 \% \mathrm{Cl}, 18.93-25.41)$ \\
\hline Mean PP Hispanic & 35.24 (95\% Cl, 26.11-44.38) \\
\hline $\begin{array}{l}\text { Mean PP African Amer- } \\
\text { ican }\end{array}$ & $52.83(95 \% \mathrm{Cl}, 45.76-59.89)$ \\
\hline $\begin{array}{l}\text { Ratio of Mean PP Hispan- } \\
\text { ic to Mean PP White }\end{array}$ & 1.59 \\
\hline $\begin{array}{l}\text { Ratio of Mean PP African } \\
\text { American to Mean PP } \\
\text { White }\end{array}$ & 2.38 \\
\hline
\end{tabular}

\section{$\operatorname{CSMR}\left(\times 10^{-4}\right)$}

\begin{tabular}{l|l}
\hline Mean CSMR White & $10.21(95 \% \mathrm{Cl}, 7.91-12.51)$ \\
\hline Mean CSMR Hispanic & $13.20(95 \% \mathrm{Cl}, 9.56-16.84)$ \\
\hline $\begin{array}{l}\text { Mean CSMR African } \\
\text { American }\end{array}$ & $37.27(95 \% \mathrm{Cl}, 30.71-43.83)$ \\
\hline
\end{tabular}

Ratio of Mean CSMR

Hispanic to Mean CSMR

White

1.29

Ratio of Mean CSMR Af-

rican American to Mean

CSMR White

\subsection{5}

\section{Statistical Analysis (Mann-Whitney U test) of PP and CSMR Data}

\section{PP Data}

Difference between daily

cumulative PP data White $P=.1091$

vs Hispanic

Difference between daily

cumulative PP data White $P<.0001$

vs African American

\section{CSMR Data}

\section{Difference between daily}

cumulative CSMR data $\quad P=.3817$

White vs Hispanic

\section{Difference between}

daily cumulative CSMR

data White vs African

$P<.0001$

American

Abbreviations: CSMR, cause-specific mortality rate; $P P$, point prevalence.

\section{DISCUSSION}

One aim in conducting this study was to determine whether there were different infection and death rates from COVID-19 among different ethnic and racial communities in Chicago during the initial COVID-19 outbreak. The other aim was to assess the implications of this information on future disease outbreaks in the United States. To achieve our first aim, we compared COVID-19 infection and death rates between White, Hispanic, and African-American Chicagoans over a 30-day period commencing at the outset of the COVID-19 infection. Our approach was to analyze the daily cumulative SARS-CoV-2 figures provided by the CDPH in order to compare SARS-CoV-2 infection and death rates between White and minority residents of the city during the initial outbreak of the infection. To directly compare the various ethnic and racial groups, we expressed these infection and death rates as the PP and the CSMR, respectively. We computed these measurements for each day of the study period, computed means, and compared these means for each racial-ethnic group in Chicago.

Figure 1 shows the daily cumulative PP of COVID-19 infections in White, African American, and Hispanic patients during the study period. The graph shows that a disproportionate number of African Americans and Hispanics compared with Whites were infected with COVID-19 during the initial outbreak. Figure 2 shows the daily cumulative CSMR during the initial disease outbreak. It shows that a significantly higher number of African American patients died during this period compares with Whites and Hispanics.

Our data can be interpreted as showing that during the initial SARS-CoV-2 outbreak, between April 5, 2020, and May 4, 2020, the rate of infection among Hispanic Chicagoans was an average of 1.59 times the rate of infection among White Chicagoans. Over the same period, the average death rate from SARS-CoV-2 among Hispanics was 1.29 times the average death rate from SARS-CoV-2 among Whites in the city. Additionally, during this period, the COVID-19 infection rate among the African American population was an average of 2.38 times the infection rate for the White population; the average death rate from SARS-CoV-2 for African Americans was 3.65 that of White Chicagoans.

Further analysis showed the differences in the daily infection rates (expressed as the daily cumulative PP) and the death rates (expressed as the daily cumulative CSMR) between African Americans and Whites was statistically significant. There were considerable differences in the PP between Whites and Hispanics and in CSMR between Whites and Hispanics. Neither of these differences reached a level of statistical significance.

We believe that the reasons for the observed differences in infection and death rates between White and minority patients may be related to long-established health disparities between White and minority populations in the United States ${ }^{12}$ : minorities 


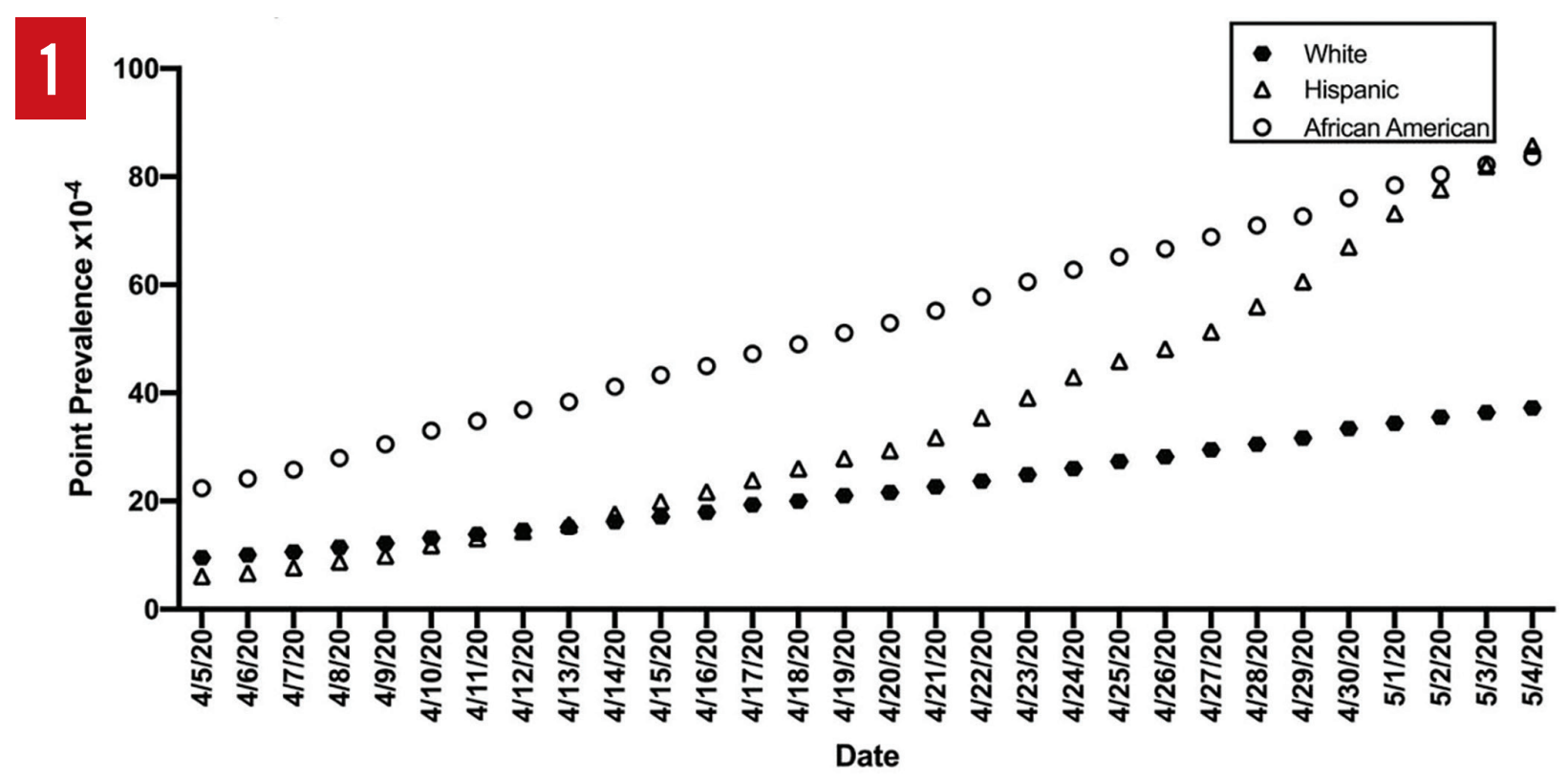

Daily Cumulative PP for White, Hispanic, and African American Patients With COVID-19
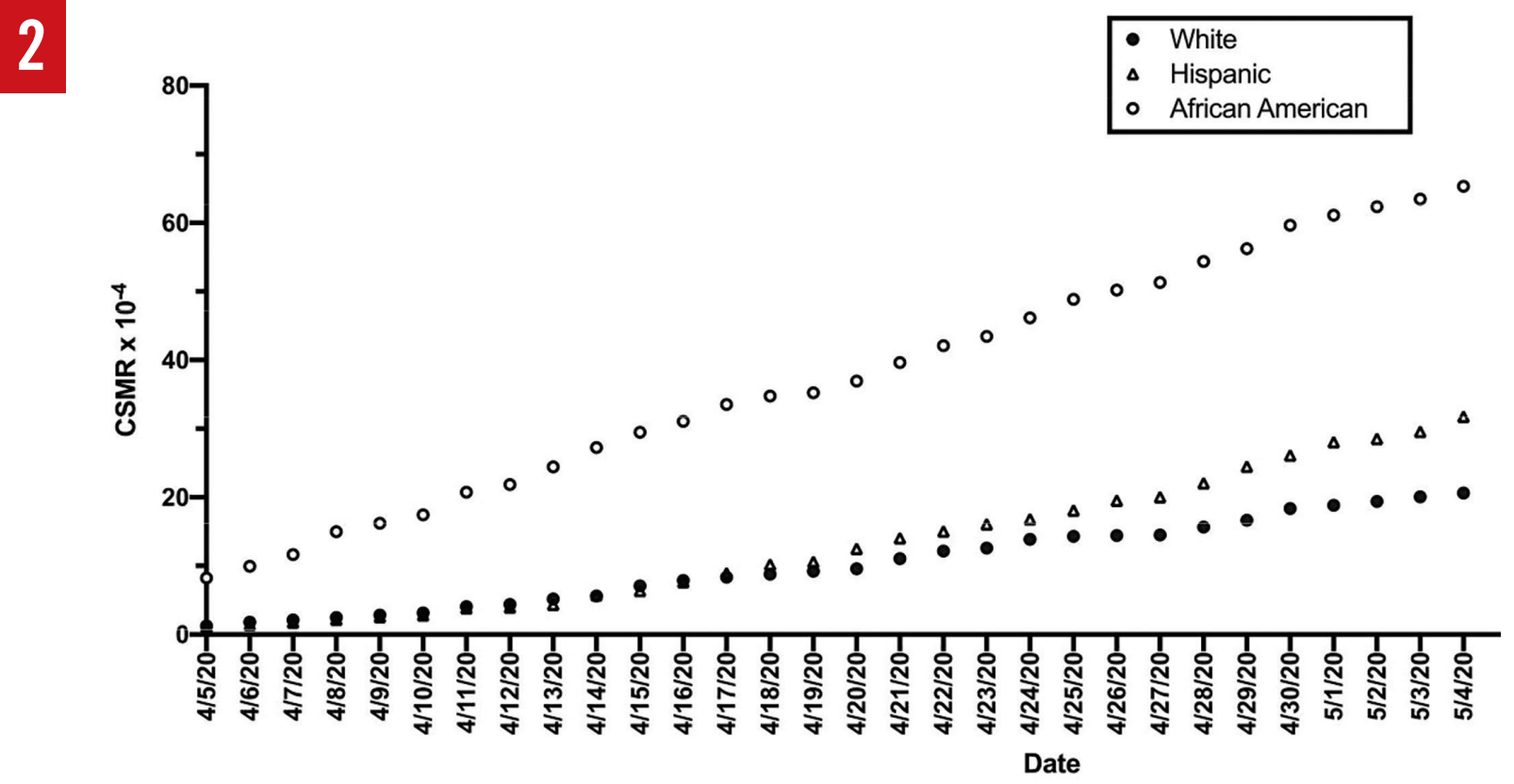

Daily Cumulative CSMR for White, Hispanic, and African American Patients With COVID-19

are known to have higher rates of comorbidity such as hypertension, obesity, diabetes, and kidney disease than White Americans. ${ }^{13-16}$ Diabetes, obesity, and kidney disease are associated with metabolic inflammation, which promotes the release of cytokines and may make the development of a cytokine storm-a possible key cause of death from SARS-CoV-2 - more likely. ${ }^{17}$
Hypertension, on the other hand, may be associated with an angiotensin receptor imbalance that predisposes patients more to severe SARS-CoV-2 infection. ${ }^{17}$ Patients with these conditions therefore may have greater SARS-CoV-2 infection rates and greater disease severity compared with healthy individuals. ${ }^{17,18}$ Additionally, at the outset of the disease in the United States, 


\section{A Population Analysis of the Initial SARS-CoV-2 Outbreak in Chicago: Implications for the Future}

rumors abounded in the African American community that Blacks had a natural immunity to SARS-CoV-2. ${ }^{19}$

Other general factors include poorer or inadequate health insurance coverage and higher rates of unemployment among minorities, ${ }^{20}$ occupations that make staying at home difficult, ${ }^{21}$ poor quality accommodations, dense living conditions, and relatively higher rates of homelessness. ${ }^{22-24}$

Our research has implications for national and local public health departments, national and local health care providers, and organizations with an interest in optimizing the health of minority populations. If minorities are known to be at a quantifiably greater risk of infection and death during the initial phases of a major disease outbreak, appropriate plans can be made and resources directed at these at-risk populations in particular to minimize the disproportionate impact of the outbreak on these population groups. ${ }^{25}$ This may be relevant should there be an anticipated second wave of SARS-CoV-2 infections, ${ }^{26}$ or should a new global pandemic emerge in the future. ${ }^{27}$

Additionally, the SARS-CoV-2 virus is known to have mutated into a new and possibly more virulent form, ${ }^{28}$ the impact of this mutation on the different ethnic populations and on the overall infection rate in the United States is as yet unknown. ${ }^{29}$

Our research has several limitations. We analyzed public data covering a 30-day period; this represents a relatively short interval in the course of the SARS-CoV-2 outbreak in Chicago. We relied entirely on the data released by the CDPH for this analysis, and we could not verify the accuracy or the completeness of this data.

Our study does not include data from other racial-ethnic minority groups in Chicago; however, we do not believe this omission has a significant impact on our conclusions. Further studies are needed to investigate the impact of racial inequalities on the spread of this new SARS virus.

\section{REFERENCES:}

1. Guo Y-R, Cao Q-D, Hong Z-S, et al. The origin, transmission and clinical therapies on coronavirus disease 2019 (COVID-19) outbreak - an update on the status. Mil Med Res. 2020;7(1):11. doi:10.1186/s40779-020-00240-0

2. Zhai P, Ding Y, Wu X, Long J, Zhong Y, Li Y. The epidemiology, diagnosis and treatment of COVID-19. Int J Antimicrob Agents. 2020;55(5):105955. doi:10.1016/j.jjantimicag.2020.105955

3. Coronavirus disease (COVID-19) pandemic. World Health Organization. Accessed September 9, 2020. https://www.who.int/emergencies/diseases/ novel-coronavirus-2019

4. Holshue ML, DeBolt C, Lindquist S, et al; Washington State 2019-nCoV Case Investigation Team. First case of 2019 novel coronavirus in the United States. N Engl J Med. 2020;382(10):929-936. doi:10.1056/ NEJMoa2001191

5. CDC COVID data tracker. Centers for Disease Control and Prevention. Updated September 9, 2020. Accessed September 9, 2020. https://covid.cdc gov/covid-data-tracker/\#cases

6. COVID-19 Dashboard by the Center for Systems Science and Engineering (CSSE) at Johns Hopkins University (JHU). Updated September 9, 2020. Accessed September 9, 2020. https://coronavirus.jhu.edu/map.html

7. City of Chicago announces first local patient with travel-related case of 2019- novel coronavirus. News release. Chicago Department of Public Health. January 24, 2020. Accessed September 9, 2020. https://www.chicago.gov/content/city/en/depts/cdph/provdrs/health_protection_and_response/news/2020/ january/city-of-chicago-announces-first-local-patient-with-travel-relate.html

8. COVID dashboard. City of Chicago. Updated September 8, 2020. Accessed September 9, 2020. https://www.chicago.gov/city/en/sites/covid-19/home/ covid-dashboard.html

9. Manyika J, Remes J, Dobbs R, Orellana J, Schaer F. Urban America: US Cities in the Global Economy. McKinsey Global Institute; April 2012:19. Accessed September 9, 2020. https://www.mckinsey.com/ /media/McKinsey/ Featured\%20Insights/Urbanization/US\%20cities\%20in\%20the\%20 global\%20economy/MGI_Urban_America_Full_Report.ashx

10. QuickFacts: Chicago city, Illinois, United States. US Census Bureau. Accessed September 9, 2020. https://www.census.gov/quickfacts/fact/table/ chicagocityillinois, US/PST045219

11. Latest data. City of Chicago. Updated September 8, 2020. Accessed September 9, 2020. https://www.chicago.gov/content/city/en/sites/covid-19/ home/latest-data.html

12. Orsi JM, Margellos-Anast H, Whitman S. Black-White health disparities in the United States and Chicago: a 15-year progress analysis. Am J Public Health. 2010;100(2):349-356. doi:10.2105/AJPH.2009.165407

13. Holmes L, Hossain J, Ward D, Opara F. Racial/ethnic variability in hypertension prevalence and risk factors in National Health Interview Survey. ISRN Hypertension. 2013;2013:257842. doi:10.5402/2013/257842

14. Hales CM, Carroll MD, Fryar CD, Ogden CL. Prevalence of obesity and severe obesity among adults: United States, 2017-2018. NCHS Data Brief. 2020;(360):1-8

15. Chow EA, Foster H, Gonzalez V, Mclver L. The disparate impact of diabetes on racial/ethnic minority populations. Clin Diabetes. 2012;30(3):130-133. doi:10.2337/diaclin.30.3.130

16. Flessner MF, Wyatt SB, Akylbekova EL, et al. Prevalence and awareness of CKD among African Americans: the Jackson Heart Study. Am J Kidney Dis. 2009;53(2):238-247. doi:10.1053/j.ajkd.2008.08.035

17. Bornstein SR, Dalan R, Hopkins D, Mingrone G, Boehm BO. Endocrine and metabolic link to coronavirus infection. Nat Rev Endocrinol. 2020;16(6):297298. doi:10.1038/s41574-020-0353-9

18. Guan W-J, Liang W-H, Zhao Y, et al. Comorbidity and its impact on 1590 patients with COVID-19 in China: a nationwide analysis. Eur Respir J. 2020; 55(5):2000547. doi:10.1183/13993003.00547-2020

19. Ross J. Coronavirus outbreak revives dangerous race myths and pseudoscience. NBC News. March 19, 2020. Accessed September 9, 2020. https:// www.nbcnews.com/news/nbcblk/coronavirus-outbreak-revives-dangerousrace-myths-pseudoscience-n1162326

20. Sohn H. Racial and ethnic disparities in health insurance coverage: dynamics of gaining and losing coverage over the life-course. Popul Res Policy Rev. 2017;36(2):181-201. doi:10.1007/s11113-016-9416-y

21. Labor force characteristics by race and ethnicity, 2018. US Bureau of Labor Statistics. October 2019. Accessed September 9, 2020. https://www.bls.gov/ opub/reports/race-and-ethnicity/2018/home.htm

22. Williams DR, Collins C. Racial residential segregation: a fundamental cause of racial disparities in health. Public Health Rep. 2001;116(5):404-416. doi:10.1093/phr/116.5.404

23. Racial inequalities in homelessness, by the numbers. National Alliance to End Homelessness. June 1, 2020. Accessed September 9, 2020. https:// endhomelessness.org/resource/racial-inequalities-homelessness-numbers/

24. Tsai J, Wilson M. COVID-19: a potential public health problem for homeless populations. Lancet Public Health. 2020;5(4):e186-e187. doi:10.1016/ S2468-2667(20)30053-0

25. Pei S, Kandula S, Shaman J. Differential effects of intervention timing on COVID-19 spread in the United States. Preprint. Posted online May 29, 2020. medRxiv. doi:10.1101/2020.05.15.20103655

26. Ali I. COVID-19: are we ready for the second wave? Disaster Med Public Health Prep. 2020;1-3. doi:10.1017/dmp.2020.149

27. Chen J. Pathogenicity and transmissibility of 2019-nCoV-A quick overview and comparison with other emerging viruses. Microbes Infect. 2020;22(2): 69-71. doi:10.1016/j.micinf.2020.01.004

28. Korber B, Fischer WM, Gnanakaran S, et al. Tracking changes in SARS CoV-2 spike: evidence that D614G increases infectivity of the COVID-19 virus. Cell. 2020;182(4):812-827.e19. doi:10.1016/j.cell.2020.06.043

29. Yao H, Lu X, Chen Q, et al. Patient-derived mutations impact pathogenicity of SARS-CoV-2. Posted online April 23, 2020. medRxiv. doi:10.1101/2020.04.14.20060160 